\title{
Use of navigation satellite signals to determine stocks of stem wood
}

\author{
Daniil Makarov ${ }^{1 *}$, Aleksandr Borisov ${ }^{2}$, Anatoly Ostylovsky ${ }^{3}$, Viktor Ivanov $^{2},{ }^{1}$, Dmitry \\ Kharlamov $^{1}$ \\ ${ }^{1}$ Federal Research Center KSC SB RAS, Krasnoyarsk, Russian Federation \\ ${ }^{2}$ Institute of Forest, KSC SB RAS, Krasnoyarsk, Russian Federation, \\ ${ }^{3}$ Siberian Federal University, Russian Federation
}

\begin{abstract}
The method for determining the stocks of stem wood using the measurement data of the linear attenuation coefficients of signals navigation satellites L1 range and general taxometric characteristics of the forest is presented. A variant of determining the stocks of stem wood by differences in contributions to the attenuation by layers of crowns and trunks is considered, taking into account the coordinate referencing of the trajectory of the probe signal in the tree stand.
\end{abstract}

\section{Introduction}

The task of restoring stocks of stem wood by remote method using navigation satellite (NS) signals has the prospect of non-contact obtaining the average value of the crosssectional area of trunks in the forest area. This indicator is determined based on the results of taxation using the regularities of the distribution of phytomass in the forest stand. The calculation of the sum of the cross-sections of the trunks is carried out according to the data of direct measurements of the diameters of the trunks at a height of $1.3 \mathrm{~m}$, their distribution by diameters per 1 hectare of the forest stand. The structure of tree crowns and trunks is physically significantly different. The wavelength of radio emission of the NS L1 range is about $19 \mathrm{~cm}$, which is much larger than the linear dimensions of the elements of the crowns - needles, leaves and small branches. The average sizes of the tree trunk diameters are in the range of $6-28 \mathrm{~cm}$. The structure of the stand forest as a mixed dielectric made of air and trees is spatially inhomogeneous in the distribution of tree elements and trees themselves, has a layered structure [1-2]. Therefore, the contributions of scattering and absorption effects to the attenuation of NS signals are different. The crown layer is semitransparent, the signal attenuation is due to the absorption of the crown elements. The volume of layer, along with absorption, is an inhomogeneous scattering medium. The study of the possibilities of using radio scanning of the forest with NS signals is an urgent task.

\section{Method of radio scanning the forest stand}

\footnotetext{
* Corresponding author: mcdan3991@yandex.ru
} 
Radioscopy of forest areas using NS signals is carried out by registering signals from navigation satellites after passing through the stand. The measurement scheme in the variant of the antenna location near the border of the forest [3] is shown in Fig. 1.

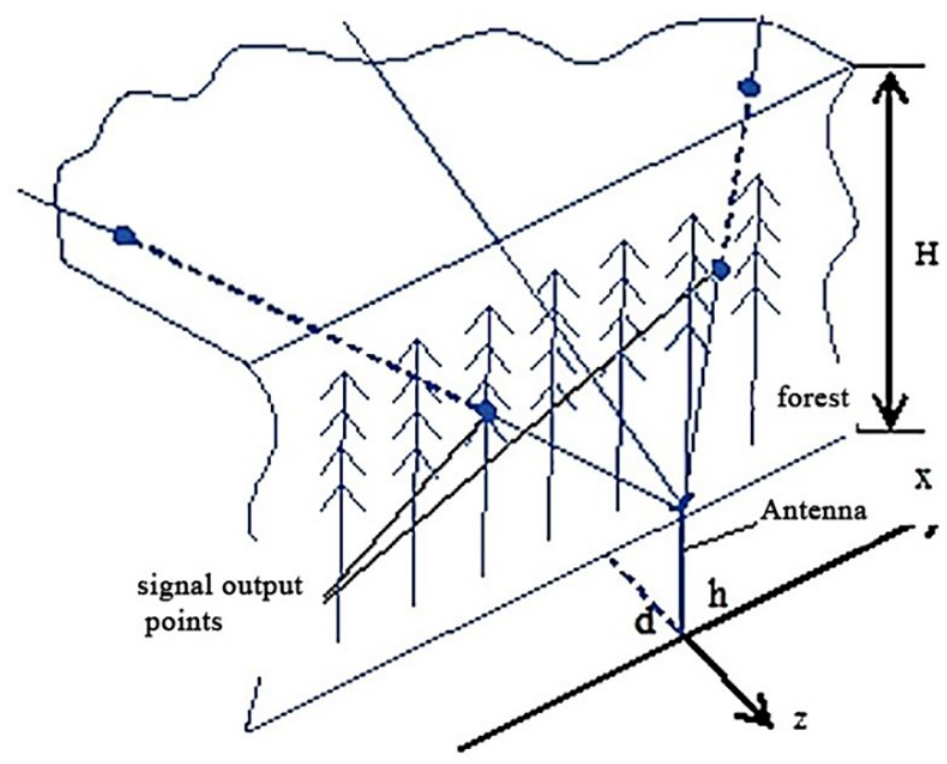

Fig 1. Scheme of the experiment on radio scanning of the tree stand.

Coordinate referencing of the signal trajectories that passed through the forest canopy makes it possible to unambiguously determine the spatial position of the analytical cone volume that forms the recorded signal. The path shown by the solid line corresponds to the registration of the direct signal that passed above the upper edge of the forest. Two paths pass through the stand in the crown layer and are indicated by dotted lines. With the change a height the antenna location $\mathrm{h}$ and its distance $\mathrm{d}$ to forest border. The contributions of crowns and trunks layers from scattering and absorption to the linear attenuation coefficients vary on different trajectories.

The method for determining the volumetric density of the forest stand biomass is based on the exponential law of attenuation of the signals of navigation satellites in the conical channel in the forest stand with the top on the receiving antenna and the solid angle corresponding to the first Fresnel zone. The antenna location in the forest stand with a vertically is oriented directional pattern axis. This allows to scan the space above the point of the antenna location in a conical volume with an angle $160^{\circ}$. Recording the probing radiation of individual satellites are realized in the analytical volume $V_{\mathrm{F}}$. This volume is determined by the magnitude $(H-h)$. The total loss of signal power during propagation in the forest is determined by absorption and multiple scattering on trunks, branches, needles or leaves.

In [1], an expression was obtained for the share of the forest stand biomass $\mu$ in the analytical volume $V_{\mathrm{F}}$, expressed in terms of the linear attenuation coefficient $\boldsymbol{v}$ of the medium and the ratio of the power of the signal falling on the stand and leaving it. The location of the analytical volume is determined unambiguously from the coordinate data of the signal trajectory and is registered by the receiver. Changing the position of the antenna 
in the crown layer makes it possible to obtain the linear attenuation coefficients on the paths in the upper part of the crown layer relative to the antenna location. As the antenna height decreases, the contribution from the upper part of the stem wood layer appears in the coefficients. In general, the bulk density of stem wood is much higher and therefore the contribution to signal attenuation will be greatest when the antenna is located near the soil surface. Analysis of the dependence of the linear attenuation coefficient on the height $h$ and taking into account the contribution from the crown layer will make it possible to determine the linear attenuation coefficient in the stem wood layer.

\section{An example of a test site}

The selection of a test site is defined by minimum number of factors affecting the interection with the NS signals. Main biometric indicators of the forest stand with small dispresy from middle are very convenient. Tree stand with small values of deviations biometric indicators from mean values are the most optimal.

This criterion is met by a even-aged pine stand based (test site) in the Pogorelsky Bor, territorially part of the Krasnoyarsk sporadic forest-steppe. The pine forest at the age of 55 is represented by a highly productive tree stand of the 1 st quality class. The spatial distribution of trees on the site is uniform, without glades and pronounced tree biogroups. Undergrowth, undergrowth and dwarf shrub layer are absent (Fig. 2).

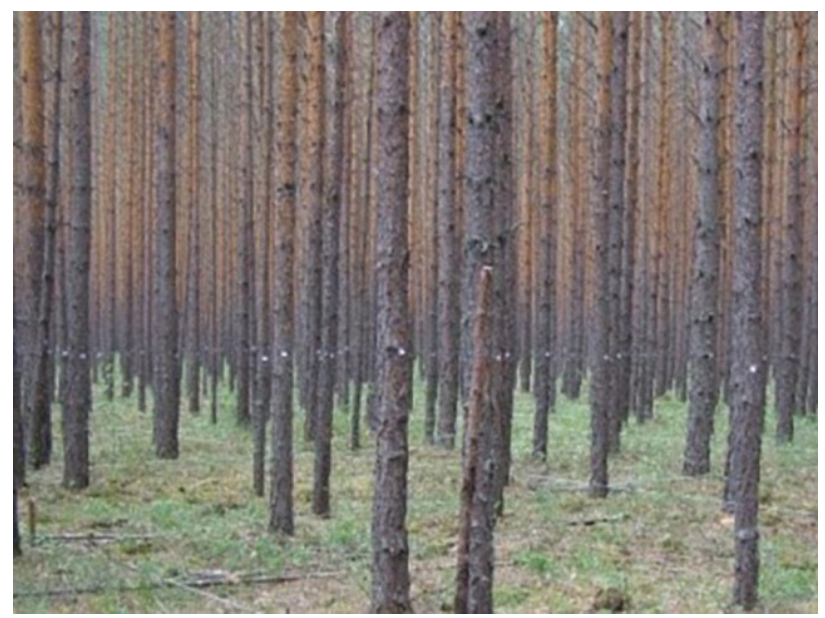

Fig 2. Arrangement of trees on the test plot: the structure of the trunk layer.

The structure of tree crowns and trunks is physically significantly different, and the parameters of the stand that affect the signal attenuation will be different. It is necessary to have two main indicators - the basal area of the trunks and the phytomass of tree crowns in the upper part to obtain more objective characteristics of signal attenuation in a plantation.

The methodology performing scientific research was used to test site [4]. The characteristics of the trees in the test site were measured. They are: the average diameter at breast height is $18.6 \mathrm{~cm}$; the average height of the stand is $20.7 \mathrm{~m}$; the average diameter of tree crowns is $2.7 \mathrm{~m}$; stand density - $1950 \mathrm{pcs} / \mathrm{ha}$; the basal area $-53 \mathrm{~m}^{2} /$ ha. The stock of stem wood on the RP calculated directly from the root area is $-514 \mathrm{~m}^{3} /$ ha. Trees data were used to calculate for other characteristics. Crown sizes vary depending on the intensity of 
competition for available resources [5]. The result of such competition on the test site is shown in Fig. 3.

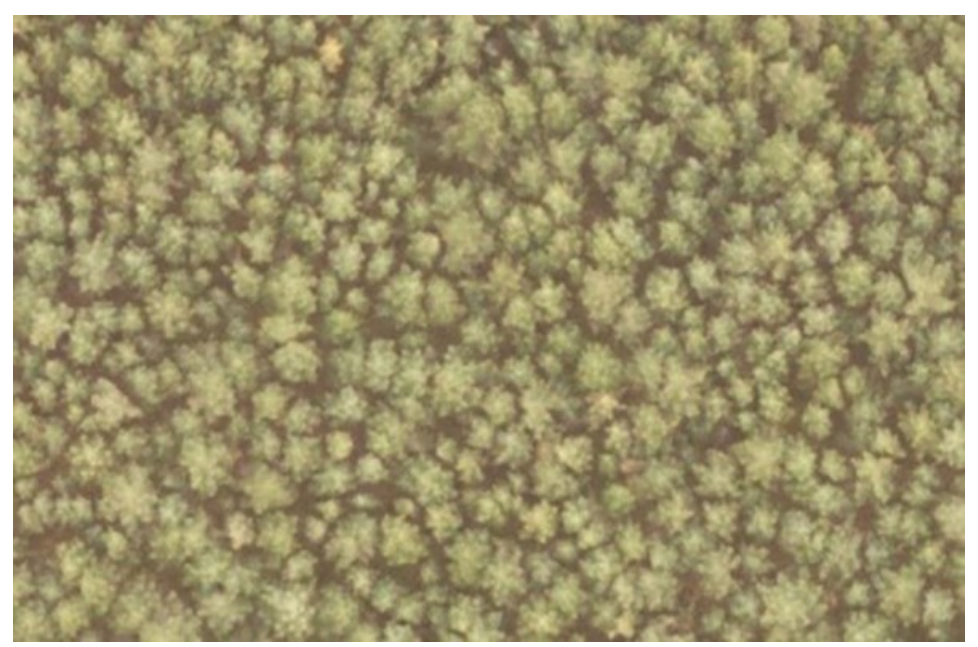

Fig 3. Arrangement of trees on the test plot: top view of the pine stand.

The size of trees by trunk diameter has significant differentiation for the same reason (Fig. 4)

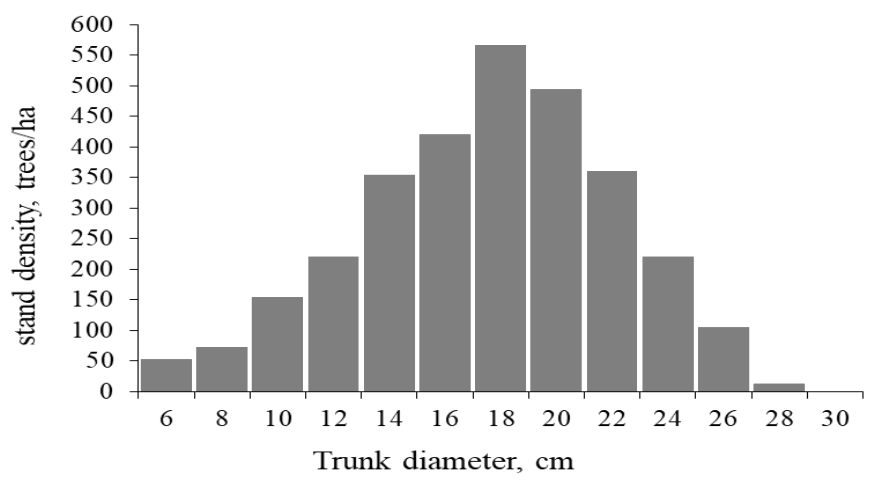

Fig 4. Distribution of trees by trunk diameter on the test site.

The ratio of crown and trunk biomass depends on the tree species, age, stand density, and available resource [6 - 8]. This ratio for sites based on Pogorelsklm Bor is about 1:6. By the height of the trees, taking into account the coefficient of the shape of the trunk, you can calculate the cross-sectional area of the trunk for a tree at any height. Taking into account the contributions of biomass components to the attenuation of the NS signal, depending on the registration angle and the antenna height, makes it possible to obtain the cross-sectional area of the trunks and the volume of stem wood in a plantation with an area of up to 3 hectares.

\section{Conclusions}


Instrumental methods for determining the stocks of stem wood using NS signals involve measuring the coefficients of linear attenuation in a representative array of the forest test site. Moving navigation satellites in the range of elevation angles from $10^{\circ}$ to $90^{\circ}$ and azimuth from $0^{\circ}$ to $360^{\circ}$ and the variable height of the antenna location allow to conduct a three-dimensional scan separately of the crown volume and the mixed volume of the crown and trunk layers.

The wavelength of the carrier radio wave in the $\mathrm{L} 1 \sim 19 \mathrm{~cm}$ range practically coincides with the average diameter of the trunks and is noticeably larger than the characteristic dimensions of individual crown elements. This circumstance allows us to separate the contributions to the coefficients of linear attenuation in the layers of crowns and trunks. An additional factor in the processes of signal attenuation is the macroscopic heterogeneity of the tree density distribution in the forest area.

Radio probing by NS signals of the forest area provides registration of space-time coordinates of the NS position, recording time, amplitude of the signal-to-noise ratio with a frequency of $1 \mathrm{~Hz}$. This information, combined with field measurements of the height of the forest, the coordinates of the location of the receiving antenna in the forest, make it possible to unambiguously determine the trajectory of the path and the linear attenuation coefficient of the NS signal in the forest.

The signal attenuation is due to the aggregate scattered on large-scale irregularities (tree trunks, large volumes of snow on the branches, irregularities in their distribution in space) and is partially absorbed in small-scale elements of trees (thin branches, needles, leaves) [911]. An additional effect on the recorded signal is made by the effect of depolarization of the GNSS signal and the appearance of a cross-polarized component due to the anisotropy of the permittivity of the forest.

The use of scanning the layered structure of the crown-stem tree stands allows one to separate their contributions to signal attenuation. The placement of the receiving antenna in the stand and taking into account the geometric characteristics of its layered structure allows one to obtain linear attenuation coefficients averaged over the volumes of crown and trunk layers. The array of obtained data has the prospect of solving the inverse problem of restoring a state of the tree stand, assessing the density of biomass and stock of stem wood.

\section{References}

1. A. Sorokin, A. Ostylovsky, A. Borisov, V. Ivanov, D. Makarov, D. Kharlamov. Assessment volume of a forest biomass by the attenuation of the navigation signals the frequencies L1. E3S Web of Conferences 149, 00 (2020) https://doi.org/10.1051/e3sconf/20201490 RPERS 20192062006

2. V. Podoprigora, A. Sorokin, D. Makarov, D. Kharlamov. Crown's anisotropy of a tree and scattering the signals of navigation satellites frequency band L1. E3S Web of Conferences 149, 00 (2020) https://doi.org/10.1051/e3sconf /20201490 RPERS 2019 2052005

3. Pat. 2682718 Russian Federation, IPC G01S 19/03 (2010.01) Method for determining the linear attenuation coefficients of navigation spacecraft signals in a forest with coordinate reference / Makarov D.S., Savin I.V., Sorokin A.V., Fomin S.V. ., Kharlamov D.V. No. 2018119448; declared 05/25/2018; publ. 03/21/2019, bull. No. 9.

4. Methodology of forest ecosystem research: methodological guide /Comp. E.N.Pilipenko, Vologda-Molochnoe, RC VGMHA, (2013)

5. O'Hara K.L. Stand structure and growing space efficiency following thinning in an even-aged Douglas-fir stand // Canadian Journal of Forest Research. Vol. 18, Pp. 859866 (1988). 
6. Stakanov V.D. The distribution of organic matter in various parts of Scots pine // Forestry,. No. 4. P.25-33. (1990)

7. Linkevicius E, Kuliesis A, Rohle H, Schroder J, Aleinikovas The Impact of Competition for Growing Space on Diameter, Basal Area and Height Growth in Pine Trees // Baltic Forestry. Vol. 20, № 2 (39). P. 301-313. (2014)

8. Nagimov V.Z., Sal'nikova I.S. Crown structure of pine trees Ural forests and forest management, Ekaterinburg, USFEU, vol. 24, pp. 161-169. (2004)

9. G.P. Karev, Structural models of forest ecosystems. Siberian Ecological Journal. 4 pp. 381-396. (1999)

10. S.N. Novik, A.S. Zavyalov, E.D. Telpukhovsky, Electrophysical and dissipative characteristics of the properties of the elements of a coniferous stand // Izvestiya Vuzov. Physics. 49, 7, P.57-67. (2006)

11. A.V. Sorokin, V.G. Podoprigora, S.V. Fomin, I.V. Savin, Evolution of the signal characteristics of navigation satellites in the forest. Izvestiya vuzov. Physics. 60. 12/2. pp. 19-22, (2017) 